\title{
¿Quién hizo qué? Diferencias entre adultos jóvenes y mayores en la memoria para un atraco
}

\author{
Alaitz Aizpurua*, Elvira García-Bajos y Malen Migueles
}

Universidad del País Vasco/Euskal Herriko Unibertsitatea

\begin{abstract}
Resumen: La probabilidad de que una persona mayor tenga que declarar como testigo o víctima de un atraco o un suceso similar aumenta en función de la esperanza de vida de la población. Por ello, resulta relevante conocer si las personas mayores producen testimonios menos exactos que los jóvenes, así como determinar cuáles son las circunstancias en las que pueden ser más proclives a cometer errores. En este estudio utilizamos una prueba de reconocimiento para analizar la memoria para las acciones de un atraco en adultos jóvenes y mayores. Aunque no hubo diferencias en los aciertos, las personas mayores cometieron una proporción superior de falsas alarmas y resultaron menos exactas que los jóvenes en el reconocimiento de las acciones del suceso. Además, las personas mayores tuvieron más dificultades que los jóvenes a la hora determinar el autor de las acciones correctamente reconocidas como pertenecientes al atraco. Por lo tanto, los déficits de las personas mayores en el control del origen de la información que recuerdan podrían ayudar a explicar la menor exactitud de su memoria para el suceso, un hallazgo especialmente relevante al tomar declaración a una persona de edad avanzada.

Palabras clave: Memoria de sucesos; envejecimiento; testigos; atribución de la fuente; juicios Recordar/Saber/Adivinar; falsas memorias.
\end{abstract}

\section{Introducción}

Con el actual aumento de la esperanza media de vida, cada vez es más probable que una persona mayor sea testigo o víctima real de un atraco, un homicidio, un accidente de tráfico u otro acontecimiento similar. Además, tenemos el estereotipo social de que las personas mayores son testigos poco fiables y esta idea preconcebida es compartida por los expertos en ámbitos policiales y judiciales (Kassin, Tubb, Hosch y Memon, 2001). Sin embargo, la investigación en el campo de la memoria de testigos se ha caracterizado por analizar la memoria de sucesos bien en niños, porque se entiende que son sugestionables y proclives a cometer errores, bien en adultos jóvenes, y realmente son muy pocos los estudios que se han centrado en examinar la memoria de las personas mayores como testigos de sucesos emocionales. Por ello, disponemos de muy poca investigación relacionada con este tema. No obstante, en la actualidad se aprecia un interés creciente general por conocer los patrones de memoria en personas de edad más avanzada, si se produce deterioro relacionado con el envejecimiento y qué recursos utilizan los adultos mayores para solventar estas posibles deficiencias.

Los delitos sobre los que tienen que declarar víctimas y testigos son acontecimientos complejos e inesperados que ocurren de forma rápida y generan un gran impacto emocional, aspectos que dificultan la memoria del suceso porque limitan la percepción completa del mismo. Estas dificultades podrían acentuarse en las personas mayores que, normal-

* Dirección para correspondencia [Correspondence address]: Alaitz Aizpurua. Facultad de Psicología, Universidad del País Vasco/Euskal Herriko Unibertsitatea. Avda. Tolosa 70, Donostia-San Sebastián, 20018. Gipuzkoa (España).E-mail: alaitz.aizpurua@ehu.es
Title: Who did what? Differences between younger and older adults in memory for a robbery

Abstract: Due to the increased life expectancy of the older population, there is a growing probability for an elderly person to testify as an eyewitness or a victim of a robbery or a similar crime. However, little is known about aging effects on testimony accuracy and about circumstances in which older adults would be more prone to memory errors than younger adults. In this study older and younger adults' memory for actions of a robbery was examined with a recognition test. Although no differences were found in hits, the older adults had a higher proportion of false alarms and showed less accurate recognition than younger adults. In addition, the older adults were less able to attribute actions that occurred during the robbery to their correct sources. Thus, source-memory deficits may contribute to explain older adults' less accurate recognition, a finding that should be taken into account in real-life eyewitness situations where an elderly person is involved.

Key words: Event memory; aging; eyewitness; source attribution; Remember/Know/Guess judgments; false memory.

mente, presentan una menor resistencia a la interferencia (Benedet, Martínez-Arias y Alejandre, 1998) y, en algunos casos, son cognitivamente menos rápidas, disponen de menos recursos atencionales y procesan la información de una forma más automática que los jóvenes (Park et al., 2002). Como resultado, por ejemplo, las personas mayores tienden a depender de la tipicidad o de la familiaridad de la información en mayor medida que los jóvenes, por lo que su probabilidad de aceptar contenidos falsos típicos es también mayor (García-Bajos, Migueles y Aizpurua, en revisión; Mather, Johnson y De Leonardis, 1999; Reder, Wible y Martin, 1986).

Normalmente para recabar información sobre un suceso se comienza pidiendo a los testigos un recuerdo libre. En general, cuando se les pide que narren la experiencia vivida, los testigos recuerdan con precisión los aspectos más representativos del suceso pero, al mismo tiempo, no son capaces de recordar todo lo ocurrido ni describen con precisión a las personas implicadas (Christianson y Hübinette, 1993; Fisher y Geiselman, 1992; Loftus, 1979; Migueles y García-Bajos, 1999; Woolnough y MacLeod, 2001; Yuille y Cutshall, 1989). Los escasos trabajos realizados con personas mayores, que simulan los delitos a través de vídeos realistas, muestran que utilizando pruebas de recuerdo libre los mayores aportan menos información que los jóvenes aunque, cuando se analiza la proporción de recuerdo erróneo, no cometen más errores que los jóvenes (Adams-Price, 1992; Aizpurua, GarcíaBajos y Migueles, 2009b, 2010; List, 1986). Las limitaciones relacionadas con el envejecimiento aparecen en el recuerdo de aspectos que requieren atención, como son los hechos específicos o detalles concretos que, no obstante, pueden ser claves para resolver casos policiales. Por lo tanto, cuando las 
personas mayores relatan libremente un suceso sus testimonios son fiables pero, en comparación con testigos más jóvenes, aportan hasta un $25 \%$ menos de información sobre las acciones, los detalles y las personas involucradas en el suceso (Aizpurua et al., 2009b, 2010).

En situaciones reales, policías, jueces y abogados también formulan a los testigos y víctimas preguntas de respuesta breve para recabar más información, o les entrevistan para verificar datos concretos. En este tipo de pruebas de reconocimiento los individuos deben discriminar entre los hechos acontecidos en el suceso y otros hechos que, aunque lógicos y coherentes con el acontecimiento, no formaban parte de la experiencia vivida. En el rendimiento de una prueba de reconocimiento normalmente se valora, por un lado, la proporción de aciertos, que se calcula en función de la cantidad de información perteneciente al suceso que se acepta como tal; por otro lado, la proporción de falsas alarmas, que es el resultado de la aceptación como verdadera de información falsa no pertenece al suceso pero compatible con el mismo; y, por último, la exactitud, que se calcula relacionando las proporciones de aciertos y falsas alarmas. En general, jóvenes y mayores reconocen correctamente hasta un $70 \%$ de los hechos del acontecimiento (Christianson y Hübinette, 1993; Hosch y Bothwell, 1990; Winningham y Weaver, 2000) pero, con frecuencia, cometen errores de memoria porque aceptan con facilidad contenidos falsos que, en base a expectativas y estereotipos, son lógicos y coherentes con la situación (Aizpurua, García-Bajos y Migueles, 2009a, 2009b, 2009c; García-Bajos y Migueles, 1999). Aunque son pocos los estudios que han analizado las diferencias relacionadas con el envejecimiento en la memoria de sucesos mediante pruebas de reconocimiento (Aizpurua et al., 2009a, 2009b, 2009c; List, 1986; Loftus, Levidow y Duensing, 1992), es en este tipo de tareas donde aparecen las mayores limitaciones derivadas del aumento de la edad. Por ejemplo, List (1986) valoró el reconocimiento de hechos ocurridos en robos a tiendas y encontró que los adultos mayores fueron menos exactos que los jóvenes; sin embargo, sólo informó de la exactitud general del reconocimiento, que calculó dividiendo los aciertos por la suma de aciertos y falsas alarmas, por lo que no podemos saber si las diferencias entre los jóvenes y mayores se encontraban en la proporción de aciertos o de falsas alarmas. En estudios más recientes se ha utilizado la Teoría de Detección de Señales, un procedimiento que permite de una forma sencilla examinar las respuestas dadas por los participantes y obtener una medida de exactitud que tiene en cuenta aciertos y falsas alarmas y una medida del criterio de respuesta. Esta última medida nos indica si una persona tiene un sesgo de respuesta conservador con una gran tendencia a decir que la información es falsa, o bien un criterio liberal o arriesgado con una tendencia a decir que la información es verdadera. En estos estudios se ha observado que no existen diferencias en los aciertos pero que las personas mayores adoptan un criterio de respuesta más liberal y aceptan la información falsa en mayor medida que los jóvenes, dando lugar a una menor exactitud; en con- creto, el nivel de falsas alarmas en lo que respecta a las acciones alcanza hasta el $45 \%$ en los jóvenes y el $60 \%$ en los mayores (Aizpurua et al., 2009a, 2009b, 2009c).

Más sorprendente que la probabilidad de los participantes de aceptar como verdadera la información falsa es el hecho de que aseguran experimentar estos errores con la misma naturaleza episódica con la que reconocen la información presentada; es decir, para ellos esos contenidos son tan reales como los recuerdos precisos. Un método muy utilizado para examinar la experiencia subjetiva de memoria es el paradigma Recordar/Saber (Tulving, 1985), en el que se distinguen los juicios recordar derivados de la memoria episódica, o autobiográfica, de las respuestas saber que se relacionan con la memoria semántica, o vinculada con los conocimientos generales. En este procedimiento se pide a los participantes que para la información que consideran verdadera indiquen si realmente recuerdan esa información, por ejemplo, porque son capaces de revivir mentalmente detalles concretos de lo ocurrido; o si, por el contrario, esa información sólo les genera una sensación de familiaridad en ausencia de la experiencia de memoria relacionada con la respuesta recordar (Gardiner, Ramponi y Richardson-Klavhen, 2002). Las falsas memorias y los aciertos reciben la misma proporción de juicios recordar (Frost, 2000). En cuanto al envejecimiento, aunque no hay diferencias en los juicios que acompañan a los aciertos, las personas mayores proporcionan más juicios recordar a sus falsas alarmas que los jóvenes y, en el caso de las acciones, aproximadamente doblan su proporción (Aizpurua et al., 2009a, 2009b, 2009c). Es decir, las personas mayores muestran una gran confianza en sus falsas memorias, un hecho que les convierte en testigos poco fiables (Dodson y Krueger, 2006).

La teoría del control de la fuente postula que en la base de estas falsas memorias está la capacidad para determinar el origen de los recuerdos, es decir, para discriminar entre lo percibido en el acontecimiento y la información sugerida o los pensamientos e inferencias elaborados con posterioridad Johnson, Hashtroudi y Lindsay, 1993; Johnson y Raye, 2000; ver también Schacter, 1996). En otras palabras, un individuo puede recordar correctamente una información pero equivocar el origen de esa información debido a que, con frecuencia, se confunden hechos percibidos con hechos activados pero que no han tenido lugar realmente. Esto ocurre, por ejemplo, cuando creemos recordar que un ladrón gritó "jesto es un atraco!" porque esta información, independientemente de si es correcta, se activa por ser típica en una situación de robo. Además, cuando los procesos implicados en el control de la fuente fracasan, podemos reconocer correctamente un elemento del pasado pero realizar una mala atribución del mismo, es decir, asignarlo a una fuente incorrecta como, por ejemplo, cuando afirmamos que alguien hizo algo y, en realidad, aunque esa acción tuvo lugar, fue otra persona quien realizó esa acción. Sin duda, analizar este aspecto es de suma importancia para la Psicología del Testimonio porque la capacidad para atribuir correctamente las 
acciones a su autor es crítica en contextos legales e, incluso, puede ser determinante a la hora de resolver un caso policial.

El objetivo principal de este estudio es examinar en el contexto de la Memoria de Testigos el rendimiento de los participantes en una tarea de atribución de la fuente, que implica la capacidad para asignar cada acción a su origen concreto, en este caso, al personaje que la realizó. Investigaciones realizadas con otros materiales revelan la existencia de cierto déficit asociado al envejecimiento en la memoria para la fuente en tareas que requieren la discriminación entre elementos presentados por una u otra persona en el laboratorio (Bayen y Murnane, 1996; Ferguson, Hashtroudi y Johnson, 1992; Hashtroudi, Johnson y Chrosniak, 1989; Kersten, Earles, Curtayne y Lane, 2008; Simons, Dodson, Bell y Schacter, 2004) y en tareas más cotidianas de la vida diaria, como identificar a la persona que dijo algo en una conversación (Brown, Jones y Davis, 1995) o al personaje literario del que procede la información (Bayen, 1999). Por ejemplo, Brown et al. (1995) simularon un grupo de conversación en el que los participantes o bien disponían de turnos para leer distintas categorías (p.e., vegetales) a responder con elementos pertenecientes a esa categoría, o bien escuchaban a otras personas intercambiar esa información y, en la prueba de memoria posterior, los adultos mayores fueron menos capaces de determinar si la categoría en cuestión había sido leída por ellos o por otra persona. No obstante, los resultados son discrepantes cuando se examina información de carácter emocional, ya que en algunos estudios se ha observado que estas diferencias se mantienen (Charles, Mather y Carstensen, 2003; Mitchell, Johnson y Mather, 2003), mientras que en otros la memoria para la fuente parece ser similar en jóvenes y mayores (May, Rahhal, Berry y Leighton, 2005; Rahhal, May y Hasher, 2002). En este experimento queremos comprobar si, en lo que a la memoria de sucesos se refiere, la capacidad para atribuir las acciones a la fuente correcta es menor en las personas mayores.

Los procesos de atribución de la fuente dependen, por un lado, de las características de la representación o huella de memoria y, por otro, del proceso de juicio implicado en la evaluación de esas características. La teoría del control de la fuente subraya que el formato de la prueba de memoria puede influir en el criterio de decisión que adopta el individuo para esta evaluación (Johnson et al., 1993; Mitchell y Johnson, 2009). Por ejemplo, listar los posibles orígenes de la información convierte al individuo en más estricto respecto a las características de la huella de memoria que posee (Johnson y Raye, 2000). Otro objetivo importante de este estudio es comprobar si el hecho de pedir explícitamente a los participantes que lleven a cabo un proceso de atribución del origen de las acciones del suceso produce una mayor exactitud en el reconocimiento que pedir la experiencia subjetiva de memoria (Recordar/Saber). Es posible que tener que hacer esta tarea de atribución permita un proceso más lento, deliberativo y racional que reduzca los errores. Analizar esta cuestión es muy relevante en el ámbito de la Psicología del Testimonio, dado que la capacidad para controlar el origen de la información recordada puede ayudar a distinguir entre un recuerdo real y un recuerdo falso.

Este tipo de experimentos únicamente se han realizado empleando el paradigma de la información engañosa o información postsuceso, un paradigma utilizado para examinar cómo incluyen los participantes en su recuerdo información falsa sugerida en una prueba de memoria posterior al suceso (ver Luna y Migueles, 2007). En estos estudios se ha encontrado que la prueba de control de la fuente reduce el efecto de la información engañosa en comparación con la prueba de reconocimiento estándar de formato sí/no o verdadero/falso (Dodson y Johnson, 1993; Lindsay y Johnson, 1989; Multhaup, De Leonardis y Johnson, 1999). Es más, Multhaup et al. (1999) encontraron que las personas mayores del grupo engañado que realizaron la prueba de reconocimiento sí/no indicaron que la información falsa sugerida en el texto formaba parte de la escena original más frecuentemente que el grupo no engañado, mientras que esta diferencia no se obtuvo entre el grupo de mayores engañado y el grupo no engañado que realizaron la tarea de control de la fuente. Es decir, la tarea de control de la fuente anuló el efecto de información engañosa en las personas mayores. Aunque este resultado es considerado como consecuencia de que los participantes que reciben la prueba de control de la fuente establecen un criterio más estricto a la hora de realizar la prueba de memoria (Dodson y Johnson, 1993; Lindsay y Johnson, 1989; Multhaup et al., 1999), sorprendentemente, en ninguno de estos estudios se informó del criterio de respuesta adoptado por los participantes al responder. Para poner a prueba esta hipótesis en el ámbito de la Memoria de Testigos, en este experimento manipulamos el tipo de tarea de memoria que pedimos a los participantes tras realizar su decisión de reconocimiento (verdadero/falso) y comparamos el rendimiento en función del tipo de tarea requerida. En base a la evidencia experimental revisada, esperamos que el hecho de pedir un recuerdo más específico de la acción inducirá a los participantes a utilizar un criterio más estricto y/o a aceptar menos acciones falsas como parte del suceso, en comparación con la condición en la que se pide la experiencia subjetiva de memoria; y, además, prevemos que esta manipulación beneficiará tanto a los adultos jóvenes como a las personas mayores, tal y como encontraron Mulhaup et al. (1999).

Por último, este experimento nos permitirá también comprobar si existen diferencias importantes entre el rendimiento de los adultos jóvenes y de las personas mayores en el reconocimiento de las acciones del suceso y, en caso afirmativo, replicar los efectos del grupo de edad que obtuvimos en estudios previos (Aizpurua et al., 2009a, 2009b, 2009c). En este sentido, podemos esperar que los adultos mayores, por un lado, establezcan un criterio de respuesta más arriesgado, acepten más acciones falsas y sean menos exactos que los jóvenes en el reconocimiento de la información. Además, prevemos que los adultos mayores proporcionarán a las falsas alarmas juicios recordar en mayor medida que los jóvenes. 


\section{Método}

\section{Participantes}

En este experimento participaron 70 adultos jóvenes (62 mujeres y 8 hombres) y 62 adultos mayores ( 37 mujeres y 25 hombres). Los adultos jóvenes, con una media de edad de 20.34 años (amplitud $=18-26$ años; $D T=3.6$ ) eran estudiantes de Educación Social de la Universidad del País Vasco y tenían una media de estudios formales de 14.43 años (amplitud $=13-19$ años; $D T=1.49)$. Los adultos mayores, con una media de edad de 64.27 años (amplitud $=56-80$ años; $D T=$ 5.32) y estudiantes de las Aulas de Experiencia de la Universidad del País Vasco, tenían una media de 14.74 años de estudios formales completados (amplitud $=11-20$ años; $D T=$ 2.65). No hubo diferencias entre jóvenes y mayores en la cantidad de años de estudios formales completados $[t(130)$ $=-.85 ; E T=.56 ; p=.40]$.

Se evaluaron las diferencias entre jóvenes y mayores en distintas habilidades cognitivas asociadas al lenguaje y la memoria, mediante la prueba de Comprensión verbal (Factor V) de la batería PMA (Thurstone y Thurstone, 1999) y la prueba de Dígitos del Wechsler Adult Intelligence Scale-III (WAIS, Wechsler, 1999). En la prueba de Comprensión verbal, que consiste en seleccionar entre cuatro alternativas propuestas un sinónimo para cada una de las cincuenta palabras, durante los tres minutos proporcionados no hubo diferencias significativas entre la media de sinónimos resueltos por los jóvenes $(M=26.56 ; D T=6.11)$ y la media de los mayores $(M=25.47 ; D T=8.11),[t(130)=.87 ; E T=1.24$; $p=.38]$. En la prueba de memoria de dígitos la cantidad de dígitos recordados en orden directo fue superior a la cantidad de dígitos recordados en orden inverso tanto en los jóvenes $[t$ $(69)=7.10 ; E T=.17]$ como en los mayores $[t(61)=7.46$; $E T=.27]$ así como en conjunto $[t(131)=9.92 ; E T=.16]$, en todos los casos $p<.001$. No hubo diferencias entre los dos grupos en el total de dígitos recordados $[M=11.76 ; D T$ $=2.11$ para jóvenes y $M=11.37 ; D T=3.08$ para mayores; $t$ $(130)=.85 ; E T=.46 ; p=.39]$. Por un lado, en la parte Digitos en orden directo, en la que los participantes deben repetir la secuencia de dígitos en el mismo orden en que se ha presentado, los jóvenes reprodujeron una media de 6.47 dígitos $(D T=1.28)$ y los mayores una media de 6.71 dígitos $(D T=$ $2)$, sin diferencias entre ambos grupos en esta medida $[t$ $(130)=-0.82 ; E T=.29 ; p=.41]$. Sin embargo, en la parte Digitos en orden inverso, en la que la secuencia debe repetirse en el orden contrario al presentado, los jóvenes $(M=5.29 ; D T$ $=1.25)$ retuvieron más dígitos que los mayores $(M=4.66$; $D T=1.75),[t(130)=2.37 ; E T=.26 ; p=.019]$. Estos resultados son comunes en la literatura relacionada con las personas mayores (p.e., Balota et al., 1999). En cualquier caso, estas medidas no correlacionaron con ningún aspecto evaluado en este experimento, ni resultaron significativas en ningún caso cuando se tomaron como covariables en los análisis de datos, por lo que podemos descartar la posibilidad de que las diferencias entre adultos jóvenes y mayores en el reconocimiento se deban a diferencias en la habilidad cognitiva de los participantes. En cambio, los resultados de estas pruebas cognitivas indican que los adultos mayores que participaron en este experimento pueden considerarse personas mayores con un buen funcionamiento cognitivo.

\section{Diseño}

Se utilizó un diseño factorial 2 (Grupo: jóvenes o mayores) x 2 (Tipo de tarea posterior al reconocimiento: juicios de memoria o atribución de la fuente) siendo ambas variables de medidas independientes. Se evaluó el rendimiento de todos los participantes mediante una prueba de reconocimiento Verdadero/Falso. Además, para las frases consideradas verdaderas, se les pidió que indicaran, en función de la condición experimental asignada, el juicio sobre la experiencia de memoria (Recordar/Saber/Adivinar) o el origen (personaje) al que atribuyen la acción. En la primera condición participaron 35 adultos jóvenes y 30 personas mayores y en la condición de atribución de la fuente participaron 35 adultos jóvenes y 32 personas mayores.

\section{Materiales}

Se presentó un suceso grabado en vídeo que mostraba un atraco de una duración aproximada de tres minutos y medio. El atraco comienza con la escena de cuatro personas, tres hombres y una mujer, en el interior de un coche que se dirige a un local de administración de lotería. En la primera parada frente a la puerta de entrada del local baja uno de ellos que entra haciéndose pasar por un cliente. Posteriormente, tras torcer la esquina del edificio, el coche para en segunda fila, quedándose el conductor en su interior. Los otros dos atracadores, un hombre y una mujer, entran a un portal que da a la parte trasera del local. Tras forzar la puerta con una palanca acceden a la parte donde están las empleadas, ambos enfundados con pasamontañas, el chico empuñando un arma y la chica con una bolsa. Al otro lado, el atracador que ha entrado primero saca una escopeta de cañones recortados y grita a los clientes que se tiren al suelo. La chica recoge el dinero que está a la vista mientras el cabecilla pide a una de las dos dependientas la llave del armario donde está el resto del dinero. Al negarse, el chico le pega un puñetazo en la cara y la mujer cae al suelo sangrando por la boca. La otra dependienta coge entonces la llave del bolsillo de la dueña y se la da a la chica, quien procede a abrir y coger el dinero del armario y después se marcha huyendo en el coche con el conductor. El cabecilla hace un gesto al compañero para que salga y seguidamente él también hace lo mismo por la puerta de atrás. Al salir, se ven abandonados sin el coche por lo que roban una moto a punta de pistola $y$ se alejan del lugar.

Primero, se establecieron dos requisitos principales a cumplir por las acciones para ser incluidas en la prueba de reconocimiento. Por un lado, las acciones verdaderas debían ser perceptivamente claras y las acciones falsas posibles y 
compatibles con el suceso. Por otra parte, se procuró que la mitad de las acciones, fueran verdaderas o falsas, tuvieran un único autor posible y la otra mitad dos posibles autores, es decir, se igualó el número de posibles atribuciones. Por ejemplo, la acción "Quedarse leyendo el periódico durante el atraco" sólo puede atribuirse a uno de los personajes implicados en el acontecimiento, en concreto, al conductor, puesto que es el único que no entra al local donde se produce el atraco y, por tanto, se considera una acción (en este caso, verdadera) con un único autor posible, es decir, de una sola atribución. En cambio, la acción "Entrar gritando jesto es un atraco!" podría haber sido realizada por cualquiera de los dos atracadores que entran al local en el momento en el que empieza el atraco y, por lo tanto, es considerada una acción (en este caso, falsa) de dos posibles atribuciones. A la hora de elaborar las acciones se intentó evitar incluir acciones ambiguas que pudieran haber sido realizadas por más de dos personajes (p.e., mirar a los lados antes de entrar al local).

En base a estos criterios se seleccionaron 20 acciones (10 verdaderas y 10 falsas). Las acciones valoraban todas las fases del acontecimiento: inicio, nudo y desenlace y recogían los hechos más representativos del suceso. La selección del material se llevó a cabo por dos jueces independientes y las discrepancias, aunque fueron escasas, se resolvieron con la ayuda de un tercer juez independiente. En el Apéndice A se presentan las acciones que se incluyeron en las pruebas de reconocimiento. Estas 20 acciones, redactadas en orden cronológico, constituyeron la prueba de reconocimiento de la que se construyeron dos modelos, uno para cada condición. En el modelo que completaron los participantes asignados a la condición de los juicios fenomenológicos, las acciones se acompañaron del sujeto de la acción (p.e., el primer atracador dice a los clientes que se callen) seguida de e los tres posibles tipos de experiencia subjetiva que podían acompañar al reconocimiento (Recordar, Saber o Adivinar). En cambio, en la prueba que completaron los participantes en la condición de atribución de la fuente, las acciones se redactaron en infinitivo (p.e., decir a los clientes que se callen) y se acompañaron de un listado con los seis posibles sujetos de la acción (el primer atracador, el conductor, el cabecilla, la chica, la dueña o la dependienta).

\section{Procedimiento}

Los participantes realizaron el experimento en dos grupos de aproximadamente cuarenta personas en el caso de los adultos jóvenes y en cuatro grupos de veinte personas en el caso de los mayores. En primer lugar, se les dijo que iban a presenciar un atraco al que debían prestar atención porque posteriormente iba a ser evaluada su memoria del suceso. La secuencia se presentó mediante un proyector de video en una pantalla de 2 x 1,5 metros. Seguidamente tras la proyección todos los participantes completaron una hoja con sus datos personales, valoraron el nivel emocional, el nivel de violencia y el nivel de impacto del suceso presentado, y se- ñalaron su experiencia previa como víctima o testigo de un suceso de este tipo.

Posteriormente se les presentó la prueba de dígitos. Primero, se les solicitó que escucharan a través de una cinta series de números y que, tras cada serie, escribieran los números en el mismo orden. Tras las 14 series, se les explicó que iban a escuchar otros 14 conjuntos de dígitos y que, en este caso, debían escribirlos en orden inverso. En uno y otro caso se les puso un ejemplo para asegurar que habían entendido la tarea. Después, los participantes dispusieron de 3 minutos para realizar la prueba de Comprensión verbal.

Finalmente, los participantes realizaron, sin límite de tiempo, la prueba de reconocimiento. Para cada una de las 20 acciones todos debían señalar si era verdadera o falsa y, en caso de considerarla verdadera, se les requirió un segundo juicio de memoria dependiendo de la condición experimental asignada. A los participantes de la condición de los juicios se les pidió que señalaran el tipo de experiencia de memoria que acompañaba a la recuperación (Recordar, Saber o Adivinar), proporcionándoles oralmente las instrucciones y algunos ejemplos para comprobar que habían entendido correctamente las diferencias entre los tres tipos de experiencia fenomenológica (ver Apéndice B). A los participantes de la condición de atribución de la fuente se les pidió, tras asegurar la correcta identificación de cada personaje (p.e., designamos dueña a la dependienta más mayor), que indicaran el sujeto que consideraban que había realizado la acción, insistiendo en que señalaran un único personaje de entre las seis alternativas posibles.

\section{Resultados}

Los participantes valoraron el suceso evaluando el nivel emocional, el nivel de violencia y el nivel de impacto del atraco en una escala tipo Likert de 7 puntos (1 bajo, 7 alto). En esta valoración, los participantes dieron una puntuación superior al nivel emocional que al nivel de violencia (5.94 vs. $5.63 ; p=.006)$ y una puntuación inferior al nivel de impacto $(M=4.92)$, con diferencias significativas entre el nivel emocional y el impacto y el nivel de violencia y el impacto $(\phi<$ .001). Además, los adultos mayores dieron al impacto puntuaciones superiores que los jóvenes $(M=5.32$ vs. 4.56; $U=$ 1484.5; $p<.001)$, sin diferencias en la valoración del nivel emocional $(M=6$ y $5.89 ; U=1956 ; p=.79)$ ni en la valoración del nivel de violencia $(M=5.79$ y $5.49 ; U=1794 ; p=$ $.08)$. Todos estos valores difirieron del valor medio 4 de la escala $(p<.001)$. Estos resultados sugieren que los participantes consideraron el suceso presenciado como violento, emocional e impactante y que este acontecimiento produjo un mayor impacto en las personas mayores que jóvenes. Estas medidas no correlacionaron con ningún aspecto evaluado en este experimento, ni resultaron significativas en ningún caso cuando se tomaron como covariables en los análisis de datos, por lo que podemos descartar la posibilidad de que las diferencias entre adultos jóvenes y mayores en el reconoci- 
miento se deban a diferencias en la valoración subjetiva del suceso.

Los participantes valoraron también su estado de salud general en una escala tipo Likert de 5 puntos que iba de 1 (muy mala) a 5 (muy buena), siendo la media para los jóvenes $4.06(D T=.68)$ y la de los mayores $4.03(D T=.62)$. No hubo diferencias entre jóvenes y mayores en esta variable $(U$ $=2122.5 ; p=.79)$, a pesar de que la mitad de las personas mayores (frente al $10 \%$ de los jóvenes) señalaron estar tomando algún medicamento.

Por otro lado, seis adultos jóvenes $(8.6 \%)$ y cinco adultos mayores $(8.1 \%)$ indicaron haber presenciado un atraco en calidad de testigos, mientras tres adultos jóvenes (4.3\%) y seis mayores $(9.7 \%)$ indicaron haber sufrido un atraco como víctimas. No obstante, estas medidas (salud, uso de medicamentos, haber sido víctima o testigo) no correlacionaron con ninguno de los aspectos valorados en este experimento ni resultaron significativas al considerarse como covariables en los análisis de datos.

\section{Reconocimiento}

Para examinar los datos del reconocimiento (véase la Tabla 1) se aplicaron cuatro análisis de varianza $(A V A R) 2 \mathrm{x}$ 2 (Grupo x Tipo de tarea) para analizar los aciertos, las falsas alarmas, la exactitud y el criterio de respuesta.

Tabla 1. Proporciones medias de aciertos, falsas alarmas, puntuaciones $A^{\prime} \mathrm{y}$ $B_{D}{ }_{D}$ en función del Grupo y del Tipo de tarea.

\begin{tabular}{|c|c|c|}
\hline & Juicios R/S/A & Atribución de la fuente \\
\hline \multicolumn{3}{|l|}{ Aciertos } \\
\hline Jóvenes & .65 & .66 \\
\hline Mayores & .69 & .69 \\
\hline \multicolumn{3}{|l|}{ Falsas alarmas } \\
\hline Jóvenes & .40 & .44 \\
\hline Mayores & .59 & .62 \\
\hline \multicolumn{3}{|l|}{$A^{\prime}$} \\
\hline Jóvenes & .67 & .64 \\
\hline Mayores & .57 & .54 \\
\hline \multicolumn{3}{|l|}{$B_{D}^{\prime \prime}$} \\
\hline Jóvenes & -.06 & -.18 \\
\hline Mayores & -.46 & -.47 \\
\hline
\end{tabular}

La proporción de aciertos fue mayor que la proporción de falsas alarmas, tanto en general $[.67$ vs. $.51 ; t(131)=9.04$; $E T=.02]$, así como cuando se solicitaron los juicios de experiencia $[.67 v$ s. $.49 ; t(64)=7.55 ; E T=.02]$ y las respuestas de atribución de la fuente $[.67$ vs. .52; $t(66)=5.42 ;$ ET $=$ .03 ], en todos los casos, $p<.001$. La proporción de aciertos también excedió la proporción de falsas alarmas en los jóvenes en general [.66 vs. .42; $t(69)=9.91 ; E T=.02]$, como en el caso de los juicios de memoria $[.65$ vs. .40; $t(34)=8.19$; $E T=.03]$ y de atribución de la fuente $[.66$ vs. $.44 ; t(34)=$ 6.01; ET $=.04]$ en particular, en todas las comparaciones, $p$ $<.001$. Y también fue así en cuanto a los mayores tanto en general $[.69$ vs. .60; $t(61)=3.48 ; E T=.02 ; p<.001]$, como en la condición de los juicios de memoria $[.69$ vs. .59; $t(29)=$
3.09; ET $=.03 ; p=.004]$. No obstante, cuando se solicitó que indicaran la fuente de la acción, la proporción de aciertos y la proporción de falsas alarmas en el grupo de las personas mayores no difirieron significativamente entre sí $[.69 \mathrm{y}$ $.62 ; t(31)=1.92 ; E T=.04 ; p=.064]$.

Aciertos. Los factores Grupo y Tipo de tarea no fueron significativos, ni tampoco la interacción entre ellos.

Falsas alarmas. En este caso únicamente resultó significativo el factor Grupo $[F(1,128)=41.29 ; M C e=.03 ; p<$ $.001]$ indicando que, en general, los mayores cometieron una mayor proporción de falsas alarmas que los jóvenes $(M=$ .60 vs. $M=.42)$.

Puntuaciones $A^{\prime}$. Las puntuaciones $A^{\prime}$ (Snodgrass y Corwin, 1988) representan la exactitud del reconocimiento. Los valores de este índice no paramétrico varían entre 0 y 1. Puntuaciones de .5 indican un rendimiento al azar, y puntuaciones superiores indican una mejor discriminación y precisión. Sólo resultó significativo el factor Grupo $[F(1,128)=$ 12.59; $M C e=.02 ; p \leq .001]$ revelando que los adultos jóvenes fueron más exactos que los mayores (.65 vs. .56) porque éstos cometieron una proporción superior de falsas alarmas que los jóvenes, dado que no hubo diferencias en los aciertos. Tanto la exactitud de los jóvenes en la tarea de los juicios $[.67$ vs. $.50 ; t(34)=6.79 ; M C e=.02 ; p<.001] \mathrm{y}$ en la tarea de atribución $[.64 v$ s. $.50 ; t(34)=5.19 ; M C e=.03 ; p<$ $.001]$, como la precisión de los mayores en la tarea de los juicios $[.57$ vs. $.50 ; t(29)=3.05 ; M C e=.02 ; p=.005]$ fue superior que el rendimiento esperado al azar. Sin embargo, el rendimiento de los mayores en la tarea de atribución de la fuente no difirió del nivel esperado por azar [.54 vs. .50; $t$ $(31)=1.55 ; M C e=.03 ; p=.13]$.

Puntuaciones B" ${ }_{D}$. Las puntuaciones B" ${ }_{\mathrm{D}}$ (Donaldson, 1992) representan el criterio de respuesta utilizado por los participantes al completar la prueba de reconocimiento. Los valores varían entre $-1 \mathrm{y}+1$. La puntuación 0 indica un criterio neutro de respuesta, los valores negativos, un criterio más liberal (tendencia a responder verdadero) y los valores positivos un criterio más estricto (tendencia a responder falso). En el criterio de respuesta también fue significativo el factor Grupo $[F(1,128)=3.9 ; M C e=28.75 ; p<.001]$. Así, los adultos mayores adoptaron un criterio de respuesta más arriesgado que los jóvenes (-.46 vs. -.12).

Tabla 2. Distribución de los juicios Recordar, Saber y Adivinar para los aciertos y las falsas alarmas en función del Grupo.

\begin{tabular}{lcc}
\hline & Jóvenes & Mayores \\
\hline Aciertos & .43 & .48 \\
Recordar & .13 & .14 \\
Saber & .09 & .07 \\
Adivinar & & \\
Falsas alarmas & .17 & .33 \\
Recordar & .09 & .16 \\
Saber & .13 & .11 \\
Adivinar & & \\
\hline
\end{tabular}




\section{Juicios Recordar, Saber y Adivinar}

Para examinar los juicios para los aciertos y las falsas alarmas (véase la Tabla 2), por un lado se realizaron dos análisis de varianza $2 \times 3$ (Grupo x Juicios) globales, uno para los aciertos y otro para las falsas alarmas y, de forma complementaria, los juicios Recordar, Saber y Adivinar se analizaron por separado para los aciertos y para las falsas alarmas mediante dos AVARes unifactoriales (Grupo).

Juicios para los aciertos. Solamente fue significativo el factor Juicios $[F(1,63)=127.94 ; M C e=.04 ; p<.001]$, indicando que los aciertos recibieron más juicios recordar que saber o adivinar $(.45 v s . .14$ y .08; $p<.001)$ y más saber que adivinar (.14 vs. .08; $p=.011)$.

Juicios para las falsas alarmas. El factor Juicios fue significativo $[F(1,63)=14.236 ; M C e=.03 ; p<.001]$, indicando que los participantes proporcionaron a las falsas alarmas una mayor proporción de juicios recordar que saber o adivinar $(.25$ vs. 12 y .12; $p<.001)$, sin diferencias entre las respuestas saber y adivinar.

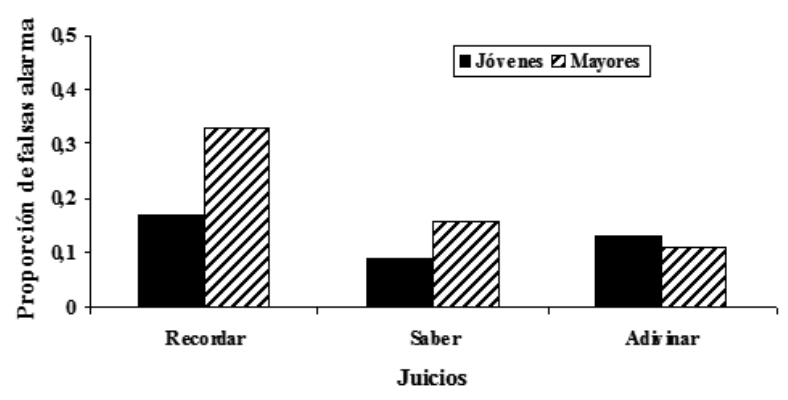

Figura 1. Distribución de los Juicios Recordar, Saber y Adivinar para las falsas alarmas en función del Grupo.
Sin embargo (ver la Figura1), tal como indicó la interacción significativa Grupo x Juicios $[F(1,63)=5.92 ; M C e=$ $.03 ;=.003]$, los mayores dieron a las falsas alarmas casi el doble de respuestas recordar en comparación con los jóvenes $(.33$ vs. $.17 ; p<.001)$ y también más respuestas saber (.16 vs. .09; $p=.041$ ) pero sin diferencias en las respuestas adivinar (.11 y .13 para mayores y jóvenes, respectivamente). Además, los mayores proporcionaron a las falsas alarmas más juicios recordar que saber $(.33 v s . .16$; $p<.001)$ o adivinar (.33 vs. $.11 ; p<.001)$, sin diferencias entre los juicios saber y adivinar; en cambio, los jóvenes dieron a las falsas alarmas más respuestas recordar que saber $(.17 \mathrm{vs} . .09 ; p=$ $.018)$, sin diferencias entre los juicios recordar y adivinar (.17 y .13) o entre los juicios saber y adivinar (.09 y .13).

\section{Atribución de la fuente}

Se analizaron por separado las atribuciones para los aciertos (Tabla 3) y para las falsas alarmas (Tabla 4).

Atribuciones para los aciertos. En el caso de los aciertos, la proporción de atribuciones correctas fue superior a la proporción de atribuciones erróneas $[.45 v$ s. .22; $t(66)=5.85$; $E T=.04 ; p<.001]$, tanto en los jóvenes [.49 vs. .17; $t(34)=$ 6.69; ET $=.05 ; p<.001]$ como en los mayores [.42 vs. .27; $t$ $(31)=2.36 ; E T=.06 ; p=.025]$.

Para los aciertos (ver la Tabla 3) se calcularon las proporciones de atribuciones correctas y erróneas y, dentro de éstas, las proporciones de atribuciones a una fuente probable y a otras fuentes. Aquellas acciones que los participantes atribuyeron a un personaje que realmente no realizó esa acción pero que podía haberla realizado se consideraron atribuciones erróneas a una fuente probable, mientras que si la atribuían a un personaje que no podía haberla realizado (p.e., por no estar presente o por estar físicamente lejos de donde se desarrolla la acción), se consideró una atribución errónea a otras fuentes.

$\underline{\text { Tabla 3. Proporciones y porcentajes de atribuciones correctas y erróneas para los aciertos. }}$

\begin{tabular}{|c|c|c|c|c|}
\hline \multirow[b]{2}{*}{ Aciertos } & \multicolumn{2}{|c|}{$\begin{array}{c}\text { Jóvenes } \\
\text { Proporción Porcentaje }\end{array}$} & \multicolumn{2}{|c|}{$\begin{array}{c}\text { Mayores } \\
\text { Proporción Porcentaje }\end{array}$} \\
\hline & .66 & 100 & .68 & 100 \\
\hline Atribución correcta & .49 & 72 & .42 & 61 \\
\hline Atribución errónea & .17 & 28 & .27 & 39 \\
\hline Fuente probable & .09 & 14 & .08 & 12 \\
\hline Otras fuentes & .08 & 14 & .19 & 27 \\
\hline
\end{tabular}

Además de las proporciones, también se calcularon los porcentajes de aciertos atribuidos a la fuente correcta, a la fuente errónea probable y a otras fuentes erróneas, porque al realizar el cálculo de las proporciones no se tiene en cuenta el número de aciertos y en el caso de los porcentajes sí y este hecho puede influir en el nivel de significación estadística de las comparaciones. Es decir, puede que la diferencia no sea estadísticamente significativa en la proporción pero sí en el porcentaje, teniendo en cuenta la cantidad de aciertos. Pos- teriormente, se realizaron comparaciones en función del Grupo en estas medidas.

Por un lado, no hubo diferencias entre jóvenes y mayores en la proporción de atribuciones correctas de los aciertos $[.49$ y $.42 ; t(65)=1.43 ; E T=.05 ; p=.16]$. Sin embargo, los jóvenes tuvieron un porcentaje superior de aciertos atribuidos a la fuente correcta que los mayores $[72 \%$ vs. $61 \% ; t(65)$ $=2.15 ; E T=.05 ; p=.035]$. Por otra parte, los mayores atribuyeron más acciones a una fuente errónea que los jóvenes $[.27$ vs. .17; $t(65)=-2.49 ; E T=.04 ; p=.015]$. Dentro de 
estas atribuciones erróneas no hubo diferencias entre jóvenes y mayores en la proporción de las acciones atribuidas a la fuente probable [.09 los jóvenes y .08 los mayores; $t(65)=$
$.89 ; E T=.02 ; p=.37]$ pero los mayores tuvieron una proporción superior de atribuciones a otras fuentes [.19 vs. .08; $t$ $(65)=-3.40 ; E T=.03 ; p=.001]$.

Tabla 4. Proporciones y porcentajes de atribuciones erróneas para las falsas alarmas.

\begin{tabular}{|c|c|c|c|c|}
\hline & \multicolumn{2}{|c|}{ Jóvenes } & \multicolumn{2}{|c|}{ Mayores } \\
\hline & Proporción & Porcentaje & Proporción & Porcentaje \\
\hline Falsas alarmas & .44 & 100 & .61 & 100 \\
\hline \multicolumn{5}{|l|}{ Atribución errónea } \\
\hline Fuente probable & .39 & 90 & .40 & 67 \\
\hline Otras fuentes & .05 & 10 & .21 & 33 \\
\hline
\end{tabular}

Atribuciones para las falsas alarmas. Para las falsas alarmas se calcularon la proporción de atribuciones erróneas a una fuente probable y a otras fuentes (ver la Tabla 4). Asimismo, se calcularon los porcentajes de falsas alarmas atribuidas a una fuente probable y a otras fuentes, porque el hecho de tener o no en cuenta el número de falsas alarmas podría influir en el nivel de significación estadística de las comparaciones. Posteriormente, se realizaron comparaciones en función del Grupo en estas medidas.

En general, la proporción de falsas alarmas atribuidas a fuentes probables fue superior que la proporción de falsas alarmas atribuidas a otras fuentes $[.40$ vs. $.13 ; t(66)=8.76$; $E T=.03 ; p<.001]$, tanto en los jóvenes $[.39$ vs. $.05 ; t(34)=$ $10.95 ; E T=.03 ; p<.001]$ como en los mayores $[.40 v s . .21 ; t$ (31) $=3.67 ; E T=.05 ; p<.001]$.

No hubo diferencias entre los adultos jóvenes y mayores en la proporción de las acciones atribuidas a una fuente probable $[.39$ y $.40 ; t(65)=-.29 ; E T=.04 ; p=.77]$ pero los jóvenes tuvieron un porcentaje superior de falsas alarmas atribuidas a una fuente probable que los mayores [90\% vs. 67\%; $t(65)=4.58 ; E T=.05 ; p<.001]$. Además, la proporción de atribuciones a otras fuentes fue superior por parte de las personas mayores que de los adultos jóvenes [0.21 vs. .05; $t$ $(65)=-5.27 ; E T=.03 ; p<.001]$.

\section{Discusión}

En este estudio examinamos el reconocimiento de las acciones de un atraco en adultos jóvenes y mayores, principalmente, para analizar las diferencias entre jóvenes y personas mayores en la capacidad para atribuir las acciones al autor en una situación de memoria de testigos y, por otro lado, para comprobar si el proporcionar la oportunidad de realizar esta tarea de control de la fuente beneficia el rendimiento de la memoria para el suceso. Tal y como esperábamos, no hubo diferencias en la proporción de aciertos pero las personas mayores cometieron una proporción superior de falsas alarmas que los jóvenes y resultaron menos exactas en el reconocimiento de las acciones del suceso. Además, las personas mayores tuvieron más dificultades que los jóvenes para determinar el autor de las acciones correctamente reconocidas como pertenecientes al atraco y, por lo tanto, estos déficits podrían explicar en parte la menor exactitud de su memoria para el suceso. No obstante, los participantes jóvenes y ma- yores que realizaron esta tarea de control de la fuente tuvieron un rendimiento muy similar al de los participantes que indicaron la experiencia subjetiva de memoria, un hallazgo que pone de manifiesto la resistencia de las falsas memorias a los efectos de la manipulación del formato de la prueba de memoria utilizada.

La capacidad para determinar quién hizo qué en una situación de memoria de testigos resulta muy importante para la exactitud del testimonio en general y, sin duda, puede ser crucial para la imputación o absolución de los acusados en un delito concreto. Los resultados del experimento que presentamos indican que, a pesar de no existir diferencias entre los adultos jóvenes y mayores en el reconocimiento de la información perteneciente al suceso (i.e., aciertos), las personas mayores mostraron una menor capacidad que los jóvenes para atribuir las acciones correctamente reconocidas a su origen. Es decir, las personas mayores fueron menos capaces que los jóvenes de señalar al autor pertinente de las acciones que realmente formaban parte del suceso. Este hallazgo es común en la literatura relacionada con el envejecimiento y la memoria de la fuente en otros contextos (p.e., Bayen y Murnane, 1996; Brown et al., 1995; Ferguson et al., 1992; Hashtroudi et al., 1989; Rahhal et al., 2002; Schacter, Kaszniak, Kihlstrom y Valdiserri, 1991; Simons et al., 2004). Sin embargo, este estudio demuestra que las limitaciones en los procesos del control de la fuente que ayudan a determinar con precisión el origen de los recuerdos también aparecen en la Memoria de Testigos.

Una buena memoria para una determinada acción requiere no sólo recordar la acción y el autor, sino también recordar qué persona desempeñó qué acción en particular; en otras palabras, requiere unir correctamente la acción con el actor correspondiente (Kersten et al., 2008). Teniendo en cuenta que las personas mayores registran la información perceptiva de manera menos distintiva que los jóvenes (p.e., DegI'Innocenti y Bäckman, 1996; Koutstaal y Schacter, 1997), y que son menos capaces de procesar la relación de un elemento y su contexto (p.e., Lyle, Bloise, \& Johnson, 2006), es lógico pensar que disponen en menor medida que los jóvenes de la información que generalmente facilita la correcta atribución de la fuente. El hecho de que las diferencias observadas entre los jóvenes y los mayores en la memoria para la fuente desaparezcan cuando se permite a las personas mayores estudiar repetidamente la información (Simons et 
al., 2004) y cuando se les insta a dirigir su atención al procesamiento de una dimensión contextual concreta (por ejemplo, la voz) que se valora (Naveh-Benjamin y Craik, 1995), apoya esta explicación. Es más, cuando los posibles orígenes de la información son perceptivamente similares, disponer de pocos detalles específicos de la información puede aumentar las dificultades que presentan las personas mayores para identificar el origen de su recuerdo (Ferguson et al., 1992; Rahhal et al., 2002).

Además, el control de la fuente no es una tarea puramente episódica sino que depende de una combinación entre procesos que recurren tanto a la información del episodio (memoria episódica) como al conocimiento preexistente (memoria semántica; Bayen, Nakamura, Dupuis y Yang., 2000; Johnson et al., 1993; Spaniol y Bayen, 2005). Las personas mayores son más proclives que los jóvenes a basar sus decisiones de reconocimiento en la familiaridad o plausibilidad de la información (Reder et al., 1986) y en los conocimientos previos (García-Bajos et al., en revisión; Mather et al., 1999) y, por lo tanto, esta predisposición a depender de la memoria semántica también podría estar implicada en su menor capacidad para la atribución de la fuente.

Muchos autores consideran que las deficiencias relacionadas con el control de la fuente son en gran medida responsables de la mayor probabilidad de reconocimiento falso que presentan las personas mayores (p.e., Balota, Dolan y Duchek, 2000; Dehon y Brédart, 2004; Norman y Schacter, 1997; ver revisiones de Schacter, Koutstaal y Norman, 1997; Spencer y Raz, 1995). Es más, la habilidad para un buen rendimiento en la tarea de control de la fuente correlaciona con la exactitud del reconocimiento, de modo que aquellos individuos que mejor realizan la tarea de atribución de la fuente muestran una mayor exactitud en la tarea de reconocimiento (Aizpurua, García-Bajos y Migueles, 2011). Por lo tanto, el déficit en lo que respecta a la memoria de la fuente de las personas mayores podría ayudar a explicar la menor exactitud de sus testimonios en el ámbito de la Memoria de Testigos.

Por otro lado, en cuanto al segundo objetivo del experimento, esperábamos encontrar que los participantes que realizaron la tarea de atribución de la fuente fueran capaces de reducir la cantidad de falsas alarmas, en base a la evidencia relacionada con las falsas memorias procedente de los estudios que revelan que la tarea de control de la fuente reduce la susceptibilidad al efecto de la información engañosa (Dodson y Johnson, 1993; Lindsay y Johnson, 1989; Multhaup et al., 1999). Sin embargo, la manipulación del tipo de tarea posterior al reconocimiento no afectó a ningún aspecto del rendimiento de los participantes. En general, encontramos un reconocimiento muy similar cuando pedimos que realizaran la tarea de control de la fuente y cuando pedimos que indicaran el juicio de memoria. Es decir, el hecho de listar los posibles orígenes de las acciones no afectó a la cantidad de aciertos ni al índice de exactitud, y tampoco influyó en la cantidad de falsas alarmas ni promovió el establecimiento de un criterio de respuesta más conservador. Este hallazgo indica que, en el campo de la Memoria de Testigos y utilizando el método del reconocimiento verdadero/falso, el requerir explícitamente a los participantes que lleven a cabo un proceso de atribución del origen de las acciones del suceso no produce efectos distintos que pedir los juicios sobre la experiencia de memoria que acompaña al reconocimiento de las mismas.

Es probable que nuestros resultados difieran de los obtenidos con el paradigma de información engañosa porque en nuestro caso manipulamos el tipo de tarea posterior al reconocimiento viejo/nuevo (control de la fuente vs. experiencia de memoria) y no el formato de la prueba de reconocimiento en sí (control de la fuente vs. reconocimiento viejo/nuevo o sí/no). Posiblemente, los participantes de nuestro estudio basaron sus decisiones de reconocimiento en el mismo tipo de información de la huella de memoria, independientemente del tipo de información que se les requirió después de tomar esa decisión (Donaldson, 1996). También puede ser que el sesgo derivado de los esquemas de conocimiento previo en la memoria para las acciones es tan intenso que no se ve afectado por el hecho de pedir a los participantes un recuerdo específico en relación al origen de la acción en el momento de la recuperación. Estudios previos muestran que la activación de esquemas y de conocimiento previo, y la memoria semántica en general, puede influir en el recuerdo de contenidos episódicos (García-Bajos y Migueles, 2003), siendo esta interacción un elemento muy poderoso en la aparición de las falsas memorias (Roediger y McDermott, 2000). En este sentido, nuestros resultados ponen de manifiesto la robustez de las falsas memorias para las acciones de un suceso independientemente del nivel de especificidad de la memoria que se requiere en la tarea posterior a la prueba de reconocimiento.

Por último, en relación a las diferencias entre adultos jóvenes y mayores en la memoria para las acciones del suceso, se replicaron los resultados previos (Aizpurua et al., 2009a, 2009b, 2009c). Así, aunque no hubo diferencias en la proporción de aciertos, las personas mayores cometieron una proporción superior de falsas alarmas que los jóvenes y resultaron menos exactas en el reconocimiento de las acciones del suceso. Los análisis realizados indicaron que las personas mayores adoptaron un criterio de respuesta más liberal que los jóvenes al responder a la prueba de memoria. Además, la proporción de falsas alarmas acompañadas de respuestas recordar fue aproximadamente el doble en las personas mayores que en los jóvenes.

\section{Conclusiones}

En 2050 aproximadamente uno de cada tres ciudadanos en España tendrá más de 65 años (Imserso, 2002). El envejecimiento de la población conlleva un aumento en la cantidad de personas mayores potenciales víctimas y testigos de diversos delitos. Por lo tanto, la investigación relacionada con la memoria en general y el recuerdo de sucesos delictivos en particular en las personas mayores es de suma importancia 
en ámbitos sociales y judiciales. Los hallazgos de este estudio sugieren que las personas mayores presentan dificultades específicas en el control del origen de la información que recuerdan, dado que la capacidad para atribuir las acciones a la persona que las realizó en el suceso fue considerablemente menor por parte de los adultos mayores que de los jóvenes. Además, independientemente de la edad del individuo, la manipulación del tipo de tarea posterior al reconocimiento (control de la fuente vs. experiencia subjetiva de memoria)

\section{Referencias}

Adams-Price, C. (1992). Eyewitness memory and aging: Predictors of accuracy in recall and person recognition. Psychology and Aging, 7, 602-608.

Aizpurua, A., García-Bajos, E. y Migueles, M. (2009a). Advertencias explícitas y falsas memorias para un suceso en adultos jóvenes y mayores. Estudios de Psicología, 30, 291-302.

Aizpurua, A., García-Bajos, E. y Migueles, M. (2009b). False memories for a robbery in young and older adults. Applied Cognitive Psychology, 23, 174187.

Aizpurua, A., García-Bajos, E. y Migueles, M. (2009c). Memory for actions of an event: Older and younger adults compared. The Journal of General Psychology, 136, 1-14.

Aizpurua, A., García-Bajos, E. y Migueles, M. (2010). Zahartzaroa eta lekukoen oroimena. Uztaro, 73, 51-68.

Aizpurua, A., García-Bajos, E. y Migueles, M. (2011). False recognition and source attribution for actions of an emotional event in older and younger adults. Experimental Aging Research, 37, 310-329.

Balota, D. A., Cortese, M. J., Duchek, J. M., Adams, D., Roedier, H. L., McDermott, K. B., et al. (1999). Veridical and false memories in healthy older adults and in dementia of the Alzheimer's type. Cognitive Neuropsychology, 16, 361-384.

Balota, D. A., Dolan, P. O. y Duchek, J. M. (2000). Memory changes in healthy older adults. En E. Tulving y F. I. M. Craik (Eds.). The Oxford Handbook of Memory (págs. 395-409). Nueva York: Oxford University Press.

Bayen, U. J. (1999). Aging and source monitoring of characters in literary texts. Aging, Neuropsychology, and Cognition, 6, 187-200.

Bayen, U. J. y Murnane, K. (1996). Aging and the use of perceptual and temporal information in source memory tasks. Psychology and Aging, 11, 293-303.

Bayen, U. J., Nakamura, G. V., Dupuis, S. E. y Yang, C. (2000). The use of schematic knowledge about sources in source monitoring. Memory \& Cognition, 18, 480-500.

Benedet, M. J., Martínez Arias, R. y Alejandre, M. A. (1998). Diferencias con la edad en el uso de estrategias, en el aprendizaje y en la retención. Anales de Psicología, 14, 139-156.

Brown, A. S., Jones, E. M. y Davis, T. L. (1995). Age differences in conversational source monitoring. Psychology and Aging, 10, 111-122.

Charles, S. T., Mather, M. y Carstensen, L. L. (2003). Aging and emotional memory: The forgettable nature of negative images for older adults. Journal of Experimental Psychology: General, 132, 310-324.

Christianson, S.Å. y Hübinette, B. (1993). Hands up! A study of witnesses' emotional reactions and memories associated with bank robberies. Applied Cognitive Psychology, 7, 365-379.

DegI'Innocenti, A. y Bäckman, L. (1996). Aging and source memory: Influences of intention to remember and associations with frontal lobe tests. Aging, Neuropsychology, and Cognition, 3, 307-319.

Dehon, H. y Brédart, S. (2004). False memories: young and older adults think of semantic associates at the same rate, but young adults are more successful at source monitoring. Psychology and Aging, 19, 191-197.

Dodson, C. S. y Johnson, M. K. (1993). Rate of false source attributions depends on how questions are asked. American Journal of Psychology, 106, 541-557.

Dodson, C. S. y Krueger, L. E. (2006). I misremember it well: Why older adults are unreliable eyewitnesses. Psychological Bulletin \& Review, 13, 770775 . no afectó a ninguna medida del reconocimiento. Ambos aspectos son relevantes para la Psicología del Testimonio y deberían ser tenidos en cuenta a la hora de tomar declaración a una víctima o testigo, especialmente si se trata de una persona de edad avanzada.

Agradecimientos.- Esta investigación se realizó gracias al proyecto PSI2012-32960 del Ministerio Español de Economía y Competitividad.

Donaldson, W. (1992). Measuring recognition memory. Journal of Experimental Psychology: General, 121, 275-277.

Donaldson, W. (1996). The role of decision processes in remembering and knowing. Memory \& Cognition, 24, 523-533.

Ferguson, S. A., Hashtroudi, S. y Johnson, M. K. (1992). Age differences in using source-relevant cues. Psychology and Aging, 7, 443-452.

Fisher, R. P. y Geiselman, R. E. (1992). Memory-enhancing techniques for investigative interviewing. The cognitive interview. Springfield, IL: Charles C. Thomas Publisher.

Frost, P. (2000). The quality of false memory over time: Is memory for misinformation "remembered" or "known"? Psychonomic Bulletin \& Review, 7, 531-536.

García-Bajos, E. y Migueles, M. (1999). Memoria de testigos en una situación emocional vs. neutra. Psicológica, 20, 91-102.

García-Bajos, E. y Migueles, M. (2003). False memories for script actions in a mugging account. European Journal of Cognitive Psychology, 15, 195-208.

García-Bajos, E., Migueles, M. y Aizpurua, A. (en revisión). Bias of scriptdriven processing on eyewitness memory in young and older adults.

Gardiner, J. M., Ramponi, C. y Richardson-Klavehn, A. (2002). Recognition memory and decision processes: A meta-analysis of remember, know, and guess responses. Memory, 10, 83-98.

Hashtroudi, S., Johnson, M. K. y Chrosniak, L. D. (1989). Aging and source monitoring. Psychology and Aging, 4, 106-112.

Hosch, H. M. y Bothwell, R. K. (1990). Arousal, description and identification accuracy of victims and bystanders. Journal of Social Behavior and Personality, 5, 481-488.

Imserso, Instituto de Migraciones y Servicios Sociales. (2002). Envejecer en España. II Asamblea Mundial sobre Envejecimiento.

Johnson, M. K., Hashtroudi, S. y Lindsay, D. S. (1993). Source monitoring. Psychological Bulletin, 114, 3-28.

Johnson, M. K. y Raye, C. L. (1981). Reality monitoring. Psychological Review, $88,67-85$.

Johnson, M. K. y Raye, C. L. (2000). Cognitive and brain mechanisms of false memories and beliefs. En D. L. Schacter y E. Scarry (Eds.), Memory, brain, and belief (págs. 35-86). Cambridge, MA: Harvard University Press.

Kassin, S. M., Tubb, V.A., T., Hosch, H. M. y Memon, A. (2001). On the "general acceptance" of eyewitness testimony research. American Psychologist, 50, 405-416.

Kersten, A. W., Earles, J. L., Curtayne, E. S. y Lane, J. C. (2008). Adult age differences in binding actors and actions in memory for events. Memory \& Cognition, 36, 119-131.

Koutstaal, W. y Schacter, D. L. (1997). Gist-based false recognition of pictures in older and younger adults. Journal of Memory and Language, 37 , 555-583.

Lindsay, D. S. y Johnson, M. K. (1989). The eyewitness suggestibility effect and memory for source. Memory \& Cognition, 17, 349-358.

List, J. (1986). Age and schematic differences in the reliability of eyewitness testimony. Developmental Psychology, 22, 50-57.

Loftus, E. F. (1979). Theoretical issues in the study of memory. En E. F. Loftus (Ed.), Eyemitness Testimony (págs. 110-133). Cambridge, M.A.: Harvard University Press.

Loftus, E. F., Levidow, B. y Duensing, S. (1992). Who remembers best? Individual differences in memory for events that occurred in a science museum. Applied Cognitive Psychology, 6, 93-107. 
Luna, K. y Migueles, M. (2007). Memoria de testigos: Patrón de distorsión de los recuerdos por la presentación de información falsa. Eguzkilore, 21, 341-363.

Lyle, K. B., Bloise, M. y Johnson, M. K. (2006). Age-related binding deficits and the content of false memories. Psychology and Aging, 21, 86-95.

Mather, M., Johnson, M. K. y De Leonardis, D. M. (1999). Stereotype reliance in source monitoring: Age differences and neuropsychological test correlates. Cognitive Neuropsychology, 16, 437-485.

May, C. P., Rahhal, T., Berry, E. M. y Leighton, E. A. (2005). Aging, source memory, and emotion. Psychology and Aging, 20, 571-278.

Migueles, M., y Garcia-Bajos, E. (1999). Recall, recognition, and confidence patterns in eyewitness memory. Applied Cognitive Psychology, 13, 257-268.

Mitchell, K. J. y Johnson, M. K. (2009). Source monitoring 15 years later: What have we learned from fMRI about the neural mechanisms of source memory? Psychological Bulletin, 135, 638-677.

Mitchell, K. J., Johnson, M. K. y Mather, M. (2003). Source monitoring and suggestibility to misinformation: Adult age-related differences. Applied Cognitive Psychology, 17, 107-119.

Multhaup, K. S., De Leonardis, D. M. y Johnson, M. K. (1999). Source memory and eyewitness suggestibility in older adults. The Journal of General Psychology, 126, 74-78.

Naveh-Benjamin, M. y Craik, F. I. M. (1995). Memory for context and its use in item memory: Comparisons of younger and older persons. Psychology and Aging, 10, 284-293.

Norman, K. A., y Schacter, D. L. (1997). False recognition in younger and older adults: Exploring the characteristics of illusory memories. Memory \& Cognition, 26, 838-848.

Park, D. C., Lautenschlager, G., Hedden, T., Davidson, N. S., Smith, A. D. y Smith, P.K. (2002). Models of visuospatial and verbal memory across adult life span. Psychology and Aging, 17, 299-320.

Pierce, B. H., Gallo, D. A. y Schacter, D. L. (2004). Aging effects on source- and gist-based false recognition. Póster presentado en Annual Meeting of Psychonomic Society, Minneapolis, MN (EEUU).

Rahhal, T. A., May, C. P. y Hasher, L. (2002). Truth and character: Sources that older adults can remember. Psychological Science, 13, 101-105.

Reder, L. M., Wible, C. y Martin, J. (1986). Differential memory changes with age: Exact retrieval versus plausible inference. Journal of Experimental Psychology: Learning, Memory, and Cognition, 12, 72-81.

Roediger III, H. L. y McDermott, K. B. (2000). Tricks of memory. Current Directions in Psychological Science, 9, 123-127.
Schacter, D. L. (1996). Searching for memory. The brain, the mind, and the past. Nueva York: BasicBooks [Traducción al castellano: En busca de la memoria. El cerebro, la mente y el pasado. Barcelona: Ediciones B., 1999].

Schacter, D. L., Kaszniak, A. W., Kihlstrom, J. F. y Valdiserri, M. (1991) The relation between source memory and aging. Psychology and Aging, 6, 559-568.

Schacter, D. L., Koutstaal, W. y Norman, K. A. (1997). False memories and aging. Trends in Cognitive Sciences, 1, 229-236.

Simons, J. S., Dodson, C. S., Bell, D. y Schacter, D. L. (2004). Specific-and partial-source memory: Effects of aging. Psychology and Aging, 19, 689694.

Snodgrass, J. G. y Corwin, J. (1988). Pragmatics of measuring recognition memory: Applications to dementia and amnesia. Journal of Experimental Psychology: General, 117, 34-50.

Spaniol, J. y Bayen, U. J. (2002). When is schematic knowledge used in source monitoring? Journal of Experimental Psychology: Learning, Memory and Cognition, 28, 631-651.

Spencer, W. D. y Raz, N. (1995). Differential effects of aging on memory for content and context: A meta-analysis. Psychology and Aging, 10, 527539.

Thurstone, L. L. y Thurstone, T. G. (1999). Aptitudes Mentales Primarias (10 ed.). Madrid: TEA Ediciones.

Tulving, E. (1985). Memory and consciousness. Canadian Psychology, 26, 1-12.

Wechsler, D. (1999). WAIS-III: Escala de inteligencia de Wechsler para adultos. Madrid: TEA Ediciones.

Winningham, R. G. y Weaver III, C. A. (2000). The effects of pressure to report more details on memories of an eyewitness event. European Journal of Cognitive Psychology, 12, 271-282.

Woolnough, P. S. y MacLeod, M. D. (2001). Watching the birdie watching you: Eyewitness memory for actions using CCTV recordings of actual crimes. Applied Cognitive Psychology, 15, 395-411.

Yuille, J. C. y Cutshall, J. I. (1989). Analysis of the statements of victims, witnesses and suspects. In J. C. Yuille (Ed.), Credibility assessment (Vol 47, págs. 175-191). Dordrecht, Netherlands: Kluwer Academic Publishers.

(Artículo recibido: 08-12-2011; revisado: 17-11-2012; aceptado: 17-11-2012) 
Apéndice A. Acciones incluidas en la prueba de reconocimiento

\author{
El conductor se queda leyendo el periódico durante el atraco \\ El cabecilla observa si hay alguien en los balcones del patio \\ El cabecilla se ajusta el pasamontañas antes de forzar la puerta \\ Y tras forzarla devuelve la palanca a la chica \\ La chica le da una pistola al cabecilla \\ $\mathrm{Y}$ éste entra gritando ¡Esto es un atraco! \\ Al otro lado, el atracador que ha entrado primero grita "iTiraros al suelo!" \\ La chica se dirige a la caja registradora \\ Apartando a la dependienta de un empujón \\ El cabecilla amenaza a la dueña apuntándole con la pistola en la cara \\ La dependienta le pide que no le haga daño \\ El primer atracador dice a los clientes que se callen \\ La dependienta coge las llaves del bolsillo a la dueña \\ La chica recoge los billetes que se han caído al suelo \\ La dependienta le ayuda a meter el dinero en la bolsa \\ El cabecilla le dice a la chica que se marche \\ Al salir la chica guarda el pasamontañas en la bolsa \\ El cabecilla amenaza a la gente al marcharse \\ El cabecilla se quita los guantes al salir \\ El primer atracador le dice al cabecilla que va a matar al conductor \\ Nota: Para la condición de atribución de la fuente, las acciones se presentaron en infinitivo.
}

Apéndice B. Instrucciones presentadas para la condición de experiencia subjetiva de memoria Cuando marques la V de verdadera deberás hacer un nuevo juicio de memoria y distinguir entre Recordar, Saber y Adivinar. A continuación explicamos las diferencias entre los juicios y damos algunos ejemplos.

$\rightarrow$ Juicios Recordar: Vas a dar juicios de Recordar cuando recuerdes detalles concretos, tengas imágenes relacionadas con la información o datos que den autenticidad a tu recuerdo.

$\rightarrow$ Juicios Saber: En este caso no dispones de datos específicos, pero la información te genera sentimientos de familiaridad, estás seguro/a de que es correcta aunque no sepas explicar por qué o dar motivos concretos sobre tu decisión.

$\rightarrow$ Juicios Adivinar: Habrá ocasiones en que no tengas ninguna experiencia de recuerdo consciente ni sentimiento alguno de familiaridad, pero pienses que no te equivocas cuando dices que es una frase verdadera.

Ejemplos

$\rightarrow$ Vas al supermercado a hacer la compra de la semana. Cuando llegas a casa te preguntan por qué no has traído uvas. Dices que estaban verdes, muy caras y eran muy pequeñas. En este caso, darías un Juicio Recordar porque tienes detalles específicos que dan validez a tu memoria y recuerdas tu reacción al verlas.

$\rightarrow$ Vas paseando por la calle y ves a una persona cuya cara se te hace conocida o familiar. Sin embargo, no tienes el recuerdo consciente de haber visto antes a esa persona. A esta situación le corresponde un Juicio Saber porque estás seguro de que no es la primera vez que ves a esa persona aunque no dispones de información específica sobre dónde, cuándo o en qué circunstancias la viste anteriormente.

$\rightarrow$ En un concurso te preguntan por la nacionalidad del escritor Frederick Forsyth. No sabes quién es pero, por el apellido, te arriesgas a decir que es inglés. La respuesta adecuada sería un Juicio Adivinar porque no recuerdas al autor ni te resulta familiar pero te arriesgas y piensas que no te equivocas al decir que es inglés. 